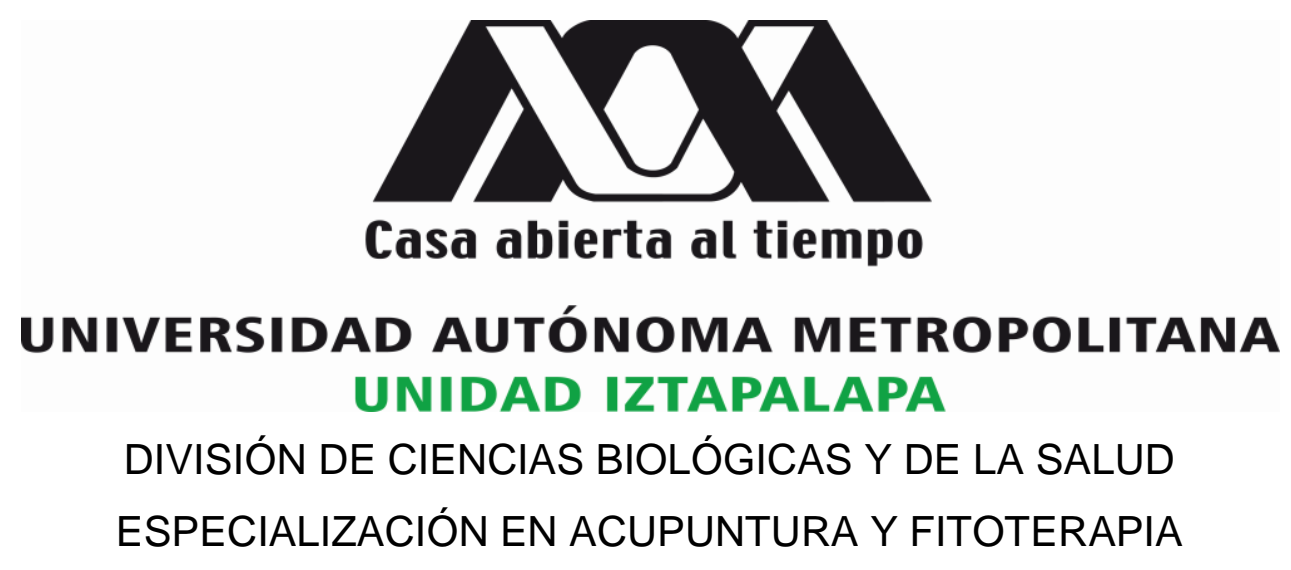

\title{
MODIFICACIÓN DE LA VARIABILIDAD DE LA FRECUENCIA CARDÍACA MEDIANTE MOXIBUSTIÓN EN EL PUNTO DE ACUPUNTURA V15 EN SUJETOS SANOS
}

\author{
IDÓNEA COMUNICACIÓN DE RESULTADOS \\ PARA OBTENER EL DIPLOMA DE LA \\ ESPECIALIZACION EN ACUPUNTURA Y FITOTERAPIA \\ Que presenta:
}

M. C. Carlos David Gordillo Paz

Director:

Dr. José Federico Rivas Vilchis

Asesor:

Dr. José Luis Eduardo Flores Sáenz

Ciudad de México, octubre de 2018 
EL JURADO DESIGNADO POR LA DIVISIÓN DE CIENCIAS BIOLÓGICAS Y DE LA SALUD DE LA UNIDAD IZTAPALAPA APROBÓ LA IDÓNEA COMUNICACIÓN DE RESULTADOS QUE PRESENTÓ:

\author{
Med. Cir. Carlos David Gordillo Paz
}

\title{
El día 8 de octubre de 2018
}

Sinodales:

Presidente:

Dr. Rubén Román Ramos

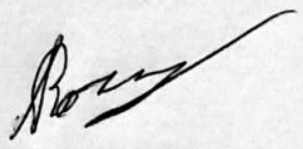

\section{Secretario:}

Dr. José Luis Eduardo Flores Sáenz

Vocal:

Dr. José Federico Rivas Vilchis

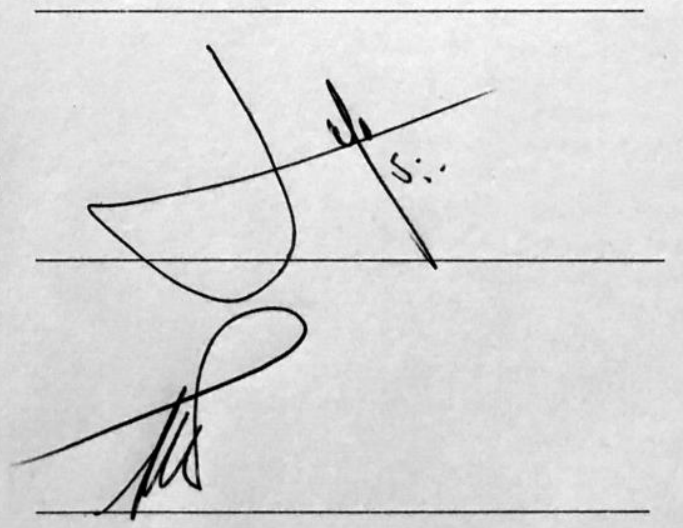




\title{
COMITÉ TUTORIAL
}

\section{DIRECTOR DE LA IDÓNEA COMUNICACIÓN DE RESULTADOS}

\author{
Dr. José Federico Rivas Vilchis \\ Profesor Titular C \\ Departamento de Ciencias de la Salud \\ Unidad Iztapalapa \\ Universidad Autónoma Metropolitana
}

\section{ASESOR DE LA IDÓNEA COMUNICACIÓN DE RESULTADOS}

Dr. José Luis Eduardo Flores Sáenz

Profesor Titular C

Departamento de Ciencias de la Salud

Unidad Iztapalapa

Universidad Autónoma Metropolitana 


\section{La presente investigación se realizó en la:}

Clínica de Acupuntura y Fitoterapia de la Universidad Autónoma Metropolitana, Unidad Iztapalapa, UAM 


\section{DEDICATORIA Y AGRADECIMIENTOS}

A Dios por nunca abandonarme.

A mi familia, en especial a mi madre Norma Evelia, por siempre apoyarme a lo largo de mi vida.

A mis maestros, en especial al Dr. José Federico Rivas Vilchis, por su paciencia y enseñanzas desde que lo conocí en la licenciatura y ahora en el Posgrado. A mis amigos, en especial a Miriam América Bonilla Anaya y Jorge Luis Alvarado Martínez por siempre alentarme a pesar de las vicisitudes 


\section{RESUMEN}

\section{Introducción}

La mayoría de los países presentan tasas elevadas y crecientes de enfermedades cardiovasculares. Estas enfermedades pueden ser evaluadas mediante la variabilidad de la frecuencia cardiaca (VFC). La VFC permite conocer el equilibrio de la actividad simpática y parasimpática del sistema nervioso autónomo (SNA). La moxibustión en el punto Vejiga 15 (V15), se sabe por experiencia clínica que modifica la fisiología y fisiopatología cardiovasculares; pero sus mecanismos no se han establecido de manera completa.

\section{Objetivo}

El objetivo de este estudio fue analizar la correlación entre la moxibustión en el punto de acupuntura V15 y los parámetros de frecuencia y tiempo de la VFC.

\section{Materiales y métodos}

Se estudiaron 14 sujetos voluntarios sanos de la comunidad de la Unidad Iztapalapa de la Universidad Autónoma Metropolitana. El estudio de la VFC se realizó utilizando el dispositivo SphygmoCor (AtCor Medical, Modelo EM3, Sydney, Australia). Se obtuvieron las variables del dominio de frecuencia y tiempo a partir del análisis espectral de intervalos sucesivos RR normales.

\section{Resultados}

La comparación basal de los parámetros de dominio y frecuencia de la variabilidad de la frecuencia cardiaca del grupo A versus grupo B mostró diferencias significativas en todos los valores del dominio de tiempo y frecuencia 
de la VFC. El tratamiento con moxibustión en V15 no originó cambios significativos en los valores de los dominios de tiempo y frecuencia de la VFC de la muestra total. En el grupo A se observaron cambios constantes pero no significativos en los valores del dominio de frecuencia de la VFC con disminución de HF e incremento de LF y LF/HF. En el grupo B no se observaron cambios significativos en los valores de la VFC. Sin embargo, los cambios originados por el tratamiento en los parámetros de tiempo y frecuencia de la VFC de los grupos A versus B tuvieron direccionalidad contraria. En el grupo A de predominio de tono parasimpático los cambios fueron en sentido de disminuir este predominio. Por el contrario, en el grupo B de predominio de tono simpático los cambios fueron en el sentido de disminuir esa orientación. Las mayores diferencias entre los grupos A y B se observaron en HF y LF. El análisis de Pearson entre las variables de tiempo y frecuencia de la VFC solo mostraron correlaciones significativas desde el punto de vista estadístico entre los valores de frecuencia en los tres grupos estudiados.

\section{Conclusión}

La estimulación del punto V15 mediante moxibustión modificó la VFC en forma diferenciada en los grupos estudiados dependiendo del estado basal del equilibrio parasimpático/simpático. En los sujetos con predominio basal parasimpático el tratamiento con moxa tendió a disminuir este predominio; mientras que en los sujetos con predominio basal simpático, se presentó un aumento de la actividad parasimpática. El tratamiento con moxibustión en V15 
modificó de manera más marcada los valores del dominio de frecuencia que los de tiempo. Consideramos que en futuros estudios en que se intente modificar la VFC con estimulación de puntos de acupuntura deberá considerarse el estado basal del equilibrio parasimpático/simpático.

Palabras clave: sistema nervioso autónomo, variabilidad de la frecuencia cardiaca, moxibustión, V15. 


\section{ABSTRACT}

\section{Introduction}

Most countries have high and increasing rates of cardiovascular diseases. These diseases can be evaluated by heart rate variability (HRV). HRV allows to know the balance of the sympathetic and parasympathetic activity of the autonomic nervous system (ANS). Is known from clinical experience that moxibustion at the Bladder 15 point (BL15) modifies cardiovascular pathophysiology; but its mechanisms have not been fully established.

\section{Objective}

The aim of this study was to analyze the correlation between moxibustion at the BL15 acupuncture point and the frequency and time values of the HRV.

\section{Materials and methods}

We studied 14 healthy volunteers from the community of Autonomous Metropolitan University, Iztapalapa campus. The study of HRV was performed using the SphygmoCor device (AtCor Medical, Model EM3, Sydney, Australia). The variables of the frequency and time domain were obtained from the spectral analysis of successive normal RR intervals.

\section{Results}

The baseline comparison of the domain and frequency parameters of the heart rate variability of group A versus group B showed significant differences in all values of the time and frequency domain of HRV. The treatment with moxibustion 
in BL15 did not originate significant changes in the values of the time and frequency domains of the HRV of the total sample. In group A, constant but not significant changes were observed in the values of the frequency domain of HRV with decreased HF and increased LF and LF/HF. No significant changes were observed in HRV values in group B. However, the changes originated by the treatment in the parameters of time and frequency of the HRV of groups A versus B had opposite directionality. In group A predominance of parasympathetic tone the changes were in the sense of decreasing this predominance. On the contrary, in group B of predominance of sympathetic tone the changes were in the sense of diminishing that orientation. The greatest differences between groups $A$ and $B$ were observed in HF and LF. The Pearson coefficient analysis between the time and frequency variables of the HRV showed only statistically significant correlations between the frequency values in the three groups studied.

\section{Conclusion}

The stimulation of the BL15 point by means of moxibustion modified the HRV in a differentiated way in the groups studied depending on the basal state of the parasympathetic/sympathetic balance. In subjects with parasympathetic basal predominance, treatment with moxa tended to decrease this predominance; whereas in subjects with sympathetic basal predominance, there was an increase in parasympathetic activity. The treatment with moxibustion in BL15 modified the values of the frequency domain in a more marked way than those of time. We consider that in future studies that attempt to modify HRV with stimulation of 
acupuncture points, the basal state of parasympathetic/sympathetic equilibrium should be considered.

Key words: autonomic nervous system, variability of heart rate, moxibustion, BL15. 


\section{PRINCIPALES ABREVIATURAS Y ACRÓNIMOS}

$\begin{array}{ll}\text { SNA } & \text { Sistema nervioso autónomo } \\ \text { V15 } & \text { Punto de acupuntura del canal Vejiga 15 } \\ \text { VFC } & \text { Variabilidad de la frecuencia cardiaca } \\ \text { RR } & \text { Intervalo RR } \\ \text { PNN50 } & \text { Porcentaje de diferencias sucesivas de NN >50 ms } \\ \text { RMSSD } & \text { Cuadrado de la raíz media de los intervalos sucesivos NN } \\ & \text { en ms } \\ \text { HF } & \text { Siglas en inglés para alta frecuencia } \\ \text { LF } & \text { Siglas en inglés para baja frecuencia }\end{array}$

Relación HF/LF Relación entre alta y baja frecuencia 


\section{ÍNDICE GENERAL}

Resumen vi

Abstract $\quad$ ix

Principales abreviaturas y acrónimos xii

$\begin{array}{ll}\text { Introducción } & 1\end{array}$

$\begin{array}{ll}\text { Preguntas de investigación } & 8\end{array}$

$\begin{array}{ll}\text { Hipótesis } & 9\end{array}$

$\begin{array}{ll}\text { Objetivos } & 10\end{array}$

$\begin{array}{ll}\text { Aspectos metodológicos } & 11\end{array}$

$\begin{array}{ll}\text { Resultados } & 15\end{array}$

$\begin{array}{ll}\text { Discusión } & 31\end{array}$

$\begin{array}{ll}\text { Limitaciones del estudio } & 38\end{array}$

$\begin{array}{lr}\text { Perspectivas } & 39\end{array}$

$\begin{array}{ll}\text { Conclusiones } & 40\end{array}$

Referencias Bibliográficas $\quad 41$

$\begin{array}{ll}\text { Anexo. } & 45\end{array}$ 


\section{INTRODUCCIÓN}

Las enfermedades cardiovasculares (ECV) son un grupo heterogéneo de enfermedades que afectan al sistema circulatorio y al corazón; destacan la arteriosclerosis, angina de pecho, hipertensión arterial, hipercolesterolemia, infarto agudo de miocardio (IAM), insuficiencias cardiacas, enfermedad cerebrovascular (ECV), trombosis arterial periférica, entre otras (Bansilal y Cols. 2015).

La mayoría de los países presentan tasas elevadas y crecientes de enfermedades cardiovasculares. Por ejemplo, en los Estados Unidos de América cada vez mueren más personas por enfermedades del corazón que de cáncer; y es la causa número uno de muerte y discapacidad en Estados Unidos y de la mayoría de países europeos. Las ECV origina uno de cada 3 decesos en el mundo; sin embargo, la mayoría de las enfermedades del corazón y el ECV son prevenibles.

En México las enfermedades no transmisibles (ENT), incluyendo enfermedades cardiovasculares, se estima que representan el $77 \%$ del total de muertes de adultos en México; y la ECV se relaciona casi una cuarta parte de estos decesos. Algunos de los factores de riesgo, relacionados con las enfermedades cardiovasculares en adultos en México, se describen a continuación:

- tabaquismo: $17 \%$ de la población es fumadora,

- alcoholismo: el consumo de alcohol se estima en 7.2 litros de alcohol puro por persona, 
- hipertensión: el $22.8 \%$ de la población tiene hipertensión, que puede aumentar el riesgo de ataque o insuficiencia cardíaca, enfermedad renal o derrame cerebral,

- obesidad: el $73 \%$ de adultos en México tienen sobrepeso u obesidad. En el año $2016,76 \%$ de las mujeres padecían de sobrepeso y obesidad en México; la obesidad es el factor de riesgo cardiovascular más común entre las mujeres (Gutierrez y Cols 2012).

\section{Variabilidad de la frecuencia cardiaca (VFC)}

La VFC es la variación de la distancia entre los intervalos RR en el electrocardiograma (ECG) y depende de fluctuaciones en la estimulación del SNA sobre el corazón, entre otros factores. En una persona sana en reposo los latidos cardiacos se producen con una frecuencia variable, es decir, los intervalos RR varían por milisegundos entre latido y latido (Draghici y Taylor 2016).

La edad, reflejo barorreceptor, respiración, temperatura y cambios posturales, entre otros factores modifican la VFC. En relación con la edad se observa que el subgrupo de adultos jóvenes muestra una elevada VFC que es una expresión del grado de ajuste vagal. Además, la VFC constituye un medio para explorar la influencia del SNA en el corazón, lo cual es un elemento predictor de mortalidad (Lees y Cols. 2018).

Los métodos espectrales se emplean de manera reciente para estudiar la VFC debido a su capacidad para separar la variabilidad del ritmo cardiaco debida a la 
excitación vagal de la producida por la excitación simpática. La utilidad de separar las dos fuentes que provocan la variabilidad del ritmo cardiaco es mejorar el diagnóstico de neuropatías y establecer el pronóstico de muerte cardiaca súbita (Benichou y Cols. 2016). Diferentes estudios han propuesto que su comportamiento depende del funcionamiento del SNA y otros sistemas de regulación fisiológicos (Gallo y Cols. 1999).

Estudios recientes han encontrado que una VFC elevada se asocia con consumo elevado de oxígeno, mientras que bajos valores en la VFC se relacionan con incremento en la mortalidad (Tsuji y Cols. 1994) y un alto riesgo en el desarrollo de patologías cardiacas en pacientes asintomáticos (Tsuji y Cols. 1994).

Métodos de medición de la VFC. De forma inicial, la VFC se evaluó manualmente a partir del cálculo del intervalo promedio R-R y su desviación estándar medida en electrocardiogramas de corta duración, por ejemplo 5 minutos. Cuanto menor es la desviación estándar en los intervalos R-R, menor es la VFC. Hasta la fecha, se informa en la literatura más de 26 tipos diferentes de manipulaciones aritméticas de los intervalos R-R para representar la VFC (Seely y Macklem 2004). La VFC puede evaluarse de dos maneras, ya sea mediante un análisis de dominio de tiempo o en el dominio de frecuencia con un análisis de densidad del espectro de potencia (DPS). En cualquier caso, se determinan primero los intervalos de tiempo entre cada complejo QRS normales sucesivos. Todos los latidos anormales no generados por las despolarizaciones del nodo sinusal se eliminan del análisis de la VFC (Rajendra y Cols. 2006). 
Análisis de tiempo. En este análisis se registran los valores de los intervalos y sus variables más comunes son:

- PNN50 (\%): es el número de intervalos adyacentes que varían por más de 50 ms expresado en porcentaje.

- RMSSD (ms): es el cuadrado de la raíz media de la unión de los intervalos R-R adyacentes; y es un indicador del control cardiaco vagal, es decir tono parasimpático (Sharabi 2009).

Análisis de frecuencia. Análisis espectral de una serie de intervalos sucesivos de $\mathrm{R}-\mathrm{R}$ que proveen el análisis del dominio de la frecuencia. Esta técnica separa el espectro de la FC en varios componentes y cuantifica influencias simpáticas y vagales sobre el corazón y son las siguientes:

- Baja frecuencia (LF, por sus siglas en inglés): son componentes que están alrededor de $0.1 \mathrm{~Hz}$. La LF depende del tono simpático a causa de la actividad de los barorreceptores (Rajendra y Cols. 2006).

- Alta frecuencia (HF, por sus siglas en inglés): componente sincronizado con la frecuencia de respiración y tiene un rango de 0.2 a $0.5 \mathrm{~Hz}$ dependiendo de la frecuencia respiratoria y es considerado un indicador de la actividad vagal (Moak y Cols. 2009).

- La relación LF/HF se considera como una imagen del equilibrio simpáticovagal o el reflejo de modulaciones simpáticas. 
En diferentes estudios clínicos y experimentales se ha podido demostrar que la actividad del sistema parasimpático es el principal responsable de los componentes de la HF, mientras que, aún no es clara la interpretación que se le da a los componentes de la LF (Rahman y Cols. 2011). Se cree que LF es un indicador sólo de la modulación simpática, aunque recientes investigaciones plantean que hace referencia a la actividad simpática y parasimpática (Goldstein y Cols. 2011).

Influyen en la VFC: la edad, el reflejo barorreceptor, la respiración, la temperatura y los cambios posturales, entre otros. En relación con la edad se observa que el subgrupo de adultos jóvenes muestra una VFC elevada que es una expresión del grado de ajuste vagal (Fatisson y Cols. 2016).

\section{Moxibustión}

La moxibustión es una forma terapéutica que forma parte de la medicina tradicional china. Los practicantes usan moxa para calentar regiones y puntos de acupuntura con la intención de estimular. El calor es generado por la combustión de una planta, la Artemisia vulgaris, de cuyo nombre en japonés, moxa, deriva el término usado en esta terapéutica. La artemisia se seca, prensa y envuelve en papel de morera, el cual tiene la capacidad de producir calor sin crear llama. La artemisia pertenece a la familia de las Composite tubuliflore. Diferentes escuelas de medicina tradicional china usan la moxa de distinta manera dependiendo del trastorno a tratar. Se enciende una de las extremidades del pequeño bastón y se 
sopla sobre éste para lograr que toda la sección sea incandescente y se consuma de forma uniforme. El extremo incandescente se aproxima al punto de acupuntura seleccionado y se cuida de mantenerlo perpendicular y a una distancia de tres a cinco centímetros de la piel aproximadamente (Noh y Cols. 2014).

\section{Punto de acupuntura V15}

El punto de acupuntura Vejiga 15 (denominado en chino Xinshu) de acuerdo a la medicina tradicional oriental regula el funcionamiento del corazón y otras funciones corporales relacionadas de manera primordial con el funcionamiento del sistema nervioso central. Se localiza a 1.5 medidas (cun) lateral a la línea media sagital, a nivel del borde inferior de la apófisis espinosa de la quinta vértebra torácica. Sus indicaciones más comunes son dolor cardiaco, palpitaciones, amnesia, irritabilidad, tos, hemoptisis, sudoración nocturna y epilepsia. Se ha reportado que diversos estímulos en V15 modifican la VFC, en especial, acupuntura, radiación electromagnética y electroacupuntura (Jun 1984).

Se ha demostrado que algunos puntos de acupuntura tienen indicaciones y efectos experimentales aparentemente antagónicos; por ejemplo el punto PC6 que se indica en algunos casos de hipertensión ha mostrado ser eficaz en disminuir las manifestaciones de hipovolemia en modelos experimentales con perros sometidos a un proceso de sangrado (Syuu y Cols. 2001). 


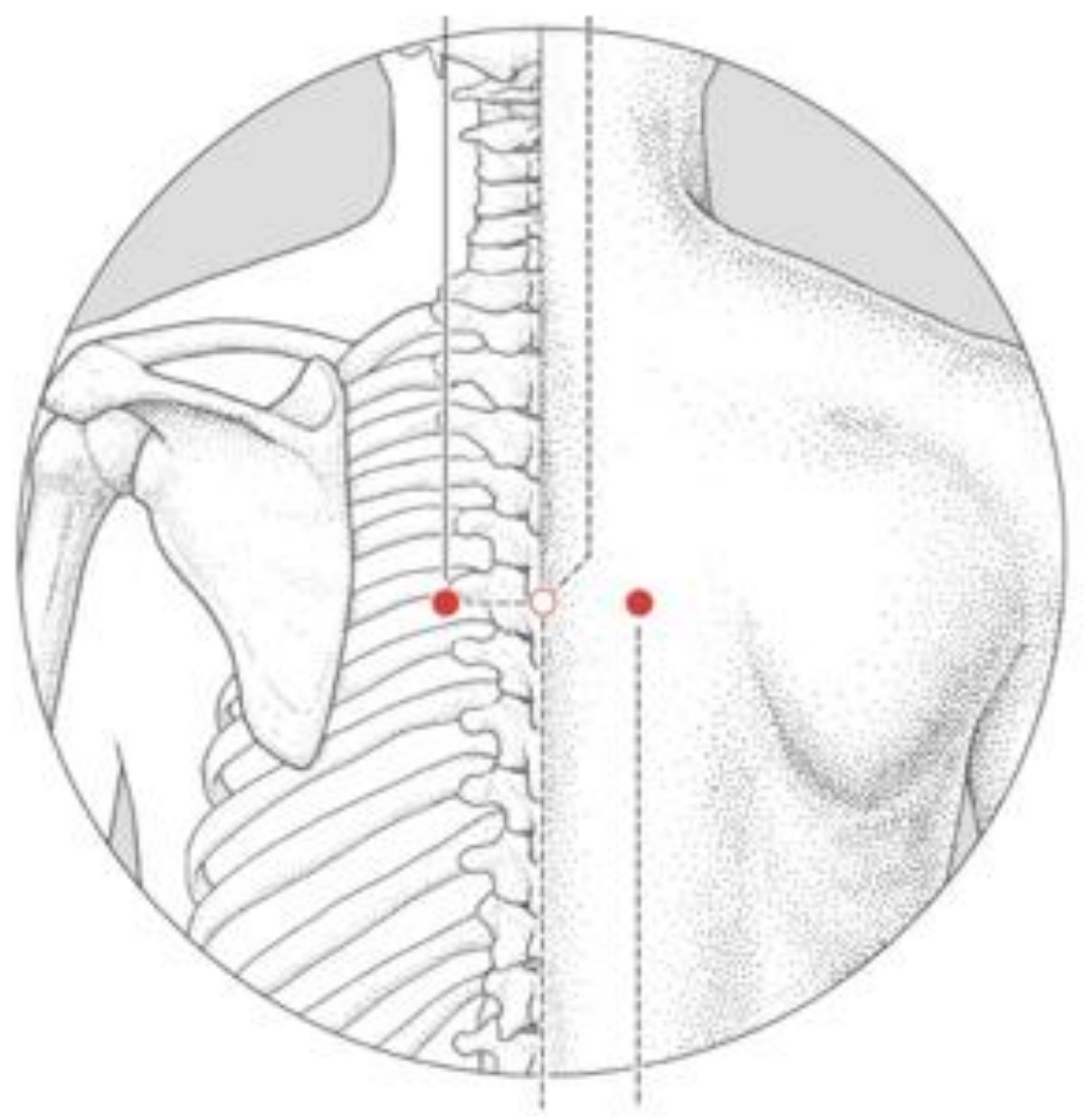

Figura 1. Punto Vejiga 15. 


\section{PREGUNTAS DE INVESTIGACIÓN}

De la información previa derivan dos preguntas experimentales:

1. ¿Produce la moxibustión indirecta en el punto V15 modificaciones en la variabilidad de la frecuencia cardiaca?

2. ¿El probable efecto de V15 dependerá de la variabilidad de la frecuencia cardiaca basal de cada sujeto? 


\section{HIPÓTESIS}

La moxibustión del punto de acupuntura V15 modifica la variabilidad de la frecuencia cardiaca en sujetos sanos de acuerdo al estado basal fisiológico cardiovascular y de actividad del sistema nervioso autónomo de cada sujeto. 


\section{OBJETIVOS}

\section{Objetivo general}

- Examinar el efecto de la moxibustión en V15 en la VFC.

\section{Objetivos específicos}

- Examinar el efecto de la moxibustión en V15 en los parámetros del dominio de frecuencia de la VFC.

- Examinar el efecto de la moxibustión en V15 en los parámetros del dominio de tiempo de la VFC.

- Analizar si existe correlación entre el efecto de la moxibustión en la VFC y el equilibrio parasimpático/simpático basal de los sujetos estudiados. 


\section{ASPECTOS METODOLÓGICOS}

Se realizó un estudio experimental de corte transversal.

\section{Población}

Los sujetos de estudio fueron reclutados en la comunidad estudiantil de la Universidad Autónoma Metropolitana.

Criterios de inclusión:

- Varones o mujeres con rango de edad entre 20 y 45 años de edad

- sanos desde el punto de vista clínico

- sin ingestión de medicamentos durante 3 días anteriores al estudio

- $\sin$ tratamiento de acupuntura durante la semana previa al estudio.

\section{Procedimiento experimental}

Se llevó a cabo la siguiente secuencia experimental:

- Toma de historia clínica abreviada

- periodo de reposo de 5 minutos

- registo de ECG basal durante 5 minutos en posición sedente

- aplicación de moxibustión

- registro de ECG pos tratamiento durante 5 minutos en posición sedente. 


\section{Variabilidad de la frecuencia cardiaca}

La variabilidad de la frecuencia cardíaca se evaluó con el participante en posición sedente. Se explicó el proceso a los participantes en forma detallada. Después de un período de reposo de 10 minutos, se registró un electrocardiograma durante 5 minutos. Los segmentos del electrocardiograma de

5 minutos se amplificaron, digitalizaron y almacenaron en una computadora, utilizando el dispositivo SphygmoCor (AtCor Medical, Modelo EM3, Sydney, Australia).

El dispositivo considera solo los latidos cardiacos normales, ignorando latidos ectópicos, para derivar la estadística parámetros de los intervalos $\mathrm{R}-\mathrm{R}$ normales (intervalos $\mathrm{N}-\mathrm{N}$ ) del electrocardiograma. Se obtuvieron las variables del dominio de frecuencia, que incluyen: alta frecuencia (HF, por sus siglas en inglés) y potencia de baja frecuencia (LF, por sus siglas en inglés) y la relación LF:HF a partir del análisis espectral de sucesivos intervalos RR (Task Force in HRV 1996; Rajendra Acharya 2006). Se empleó el método autorregresivo para evaluar el poder de espectro de densidad de las series $\mathrm{NN}$ en los tres periodos estudiados de 5 min, ver Figura 2. 


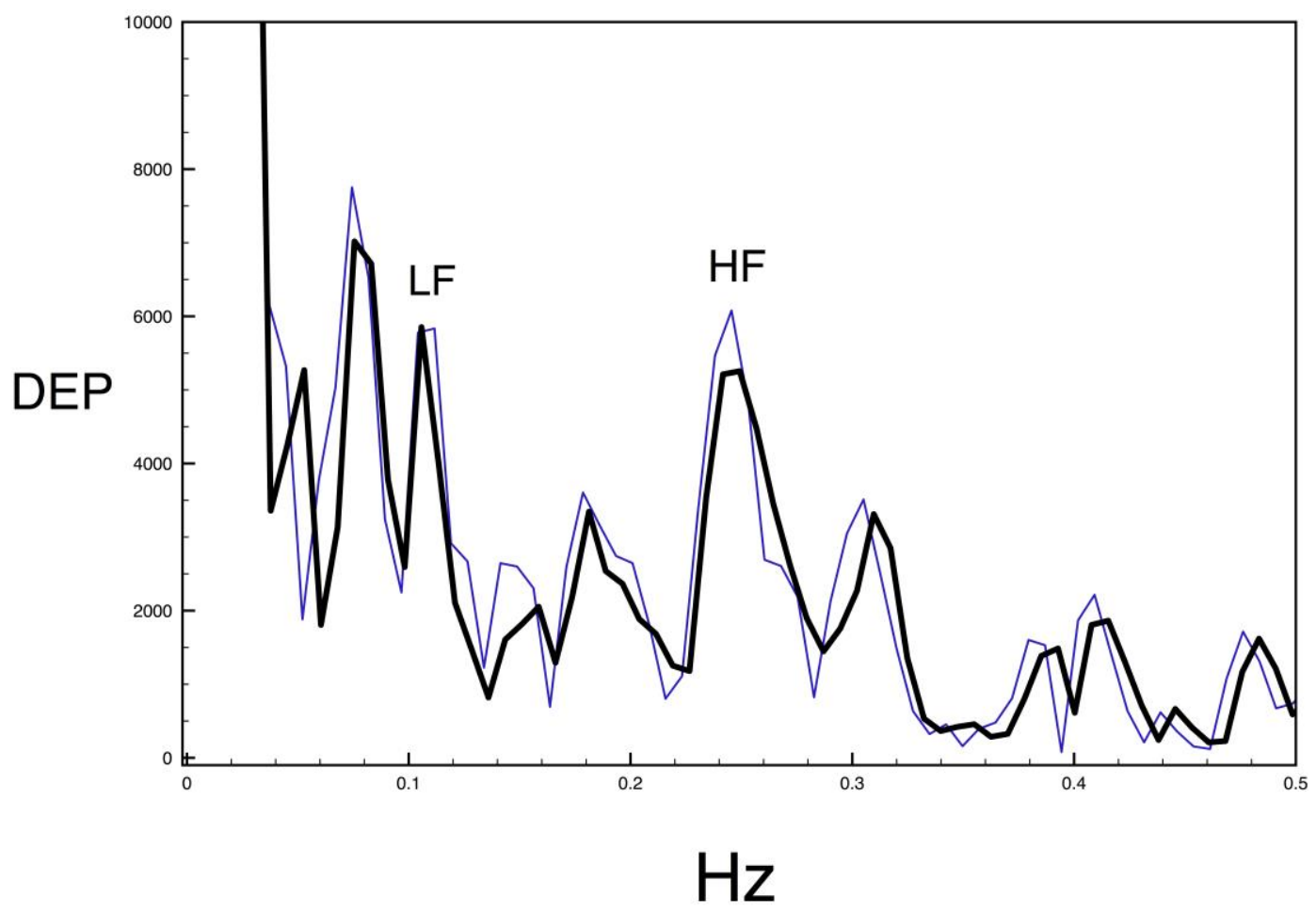

Figura 2. Registro típico de la densidad del espectro de potencia (DEP) de la variabilidad de la frecuencia cardiaca. LF, baja frecuencia y HF, baja y alta frecuencia por sus siglas en inglés, de manera respectiva.

\section{Tratamiento con moxibustión}

Se aplicó moxibustión indirecta con puros de moxa hechos a base de artemisa y se estimuló el punto V15 a una distancia de $1.0-1.5 \mathrm{~cm}$ durante un min. El punto de acupuntura Vejiga 15 se localiza a $3.5 \mathrm{~cm}$ aproximadamente lateral a la línea media dorsal y al nivel del borde inferior de la apófisis espinosa de la quinta vértebra torácica.

Constitución de los grupos A y B 
Después de la determinación de los valores de los dominios de tiempo y frecuencia se constituyeron dos grupos: el primero denominado A con predominio de actividad parasimpática con combinación de valores elevados de HF y bajos de LF y grupo B con predominio de actividad simpática con valores bajos de HF y elevados de LF.

\section{Análisis estadístico}

Los datos se reportan como media \pm desviación estándar. Se compararon los datos mediante la prueba $t$-student para datos pareados para las comparaciones intragrupo y datos no pareados para comparaciones intergrupo mediante el software Origin v. 5.0 (OrigenLab, Northampton, MA, USA). Se empleó el coeficiente de correlación de Pearson para estudiar las asociaciones entre las variables.

\section{Consideraciones éticas}

Se cumplieron los preceptos legales para la investigación en seres humanos de la legislación mexicana y las recomendaciones internacionales aplicables (Declaration of Helsinki, 2013). Se explicó en detalle los propósitos y método de la investigación a cada participante y aquellos que aceptaron participar firmaron una declaración de consentimiento informado.

\section{RESULTADOS}

\section{Población estudiada}


Las características de los grupos estudiados: total, grupo A con predominio de actividad simpática y grupo B con predominio de actividad parasimpática se muestran en los Cuadros 1, 2 y 3 de manera respectiva.

Cuadro 1. Características demográficas de la muestra total.

\begin{tabular}{ccc} 
Variable & $n$ & Edad $^{*}$ \\
\hline Total & 14 & $28.1 \pm 6.3$ años \\
\hline Varones & $8(57.2 \%)$ & $29.5 \pm 6.9$ años \\
\hline Mujeres & $6(42.8 \%)$ & $26.3 \pm 5.5$ años
\end{tabular}

*Media \pm desviación estándar.

Cuadro 2. Características demográficas de los grupos $A$ con tono parasimpático predominante y $\mathrm{B}$ con tono simpático predominante.

\begin{tabular}{lcc}
\hline Variables & Grupo A & Grupo B \\
\hline $\begin{array}{l}\text { Mujeres } \\
\mathrm{n}(\%)\end{array}$ & $3(42.8 \%)$ & $3(42.8 \%)$ \\
\hline $\begin{array}{l}\text { Edad } \\
(\mathrm{n} \pm \mathrm{DE})\end{array}$ & $27.7 \pm 5.8$ años & $25 \pm 6.1$ años \\
\hline $\begin{array}{l}\text { Varones } \\
\mathrm{n}(\%)\end{array}$ & $4(57.2 \%)$ & $4(57.2 \%)$ \\
\hline $\begin{array}{l}\text { Edad } \\
(\mathrm{m} \pm \mathrm{DE})\end{array}$ & $27.3 \pm 5.7$ años & $31.8 \pm 8.1$ años \\
Media \pm desviación estándar. & & \\
\hline
\end{tabular}

\section{Comparación entre el grupo A y el grupo B}

Las comparaciones de las variables de dominio de tiempo y de frecuencia de la VFC entre el grupo A de predominio tono parasimpático y grupo $B$ de predominio 
tono simpático en condiciones basales se muestran en los cuadros 3 y 4 , de manera respectiva.

Cuadro 3. Comparación de la variabilidad de la frecuencia cardiaca en los grupos A de predominio parasimpático y el B de predominio simpático en condiciones basales.

\begin{tabular}{lccc}
\hline & $\begin{array}{c}\text { Grupo A } \\
\text { (media } \pm \text { DE) }\end{array}$ & $\begin{array}{c}\text { Grupo B } \\
\text { (media } \pm \text { DE) }\end{array}$ & $p$ \\
\hline Dominio de tiempo & & & \\
\hline PNN50 & $33.7 \pm 5.8$ & $26.9 \pm 3.7$ & 0.0229 \\
\hline RMSSD & $61.1 \pm 3.7$ & $52.1 \pm 7.9$ & $0.0178^{*}$ \\
\hline
\end{tabular}

\section{Dominio de frecuencia}

\begin{tabular}{lccl}
\hline $\mathrm{HF}$ & $43.5 \pm 21.8$ & $20.7 \pm 3.2$ & $0.0180^{\star}$ \\
\hline $\mathrm{LF}$ & $56.5 \pm 21.8$ & $79.3 \pm 3.2$ & $0.0180^{\star}$ \\
\hline LF/HF & $1.8 \pm 1.2$ & $4.0 \pm 1.0$ & $0.0034^{\star}$
\end{tabular}

PNN50: número de intervalos adyacentes que varían por más de $50 \mathrm{~ms}$ expresado en porcentaje.

RSSMD: cuadrado de la raíz media de la unión de los intervalos $R-R$ adyacentes.

HF: alta frecuencia. LF: baja frecuencia. Relación HF/LF: relación entre alta y baja frecuencia.

DE: desviación estándar.

$\mathrm{P}=$ valor $\mathrm{p}$ de la prueba de $t$-student.

${ }^{*} \mathrm{p}<0.05$

Se observa que los parámetros de dominio y frecuencia de la variabilidad de la frecuencia cardiaca del grupo A versus grupo B mostraron diferencias significativas en todos los casos. 


\section{Efecto del tratamiento con moxibustión en la muestra total y los grupos A y B}

Grupo total. El efecto de tratamiento con moxibustión en el grupo total se muestra en el Cuadro 4.

Cuadro 4. Efecto del tratamiento con moxibustión en el punto V15 en la variabilidad de la frecuencia cardiaca en la población total.

\begin{tabular}{lccc}
\hline & $\begin{array}{c}\text { Basal } \\
(\text { media } \pm \text { DE })\end{array}$ & $\begin{array}{c}\text { Tratado } \\
(\text { media } \pm \text { DE })\end{array}$ & P \\
\hline Dominio de tiempo & & & \\
\hline PNN50 & $30.3 \pm 5.8$ & $30.2 \pm 9.7$ & 0.9370 \\
\hline RMSSD & $56.6 \pm 7.5$ & $56.7 \pm 11.1$ & 0.9483 \\
\hline
\end{tabular}

Dominio de frecuencia

\begin{tabular}{lccc}
\hline HF & $32.1 \pm 19.1$ & $29.5 \pm 13.5$ & 0.3416 \\
\hline LF & $67.9 \pm 19.1$ & $70.5 \pm 13.5$ & 0.3416 \\
\hline LF/HF & $2.9 \pm 1.5$ & $2.92 \pm 1.4$ & 0.9227
\end{tabular}

PNN50: número de intervalos adyacentes que varían por más de $50 \mathrm{~ms}$ expresado en porcentaje. RSSMD: cuadrado de la raíz media de la unión de los intervalos $\mathrm{R}-\mathrm{R}$ adyacentes.

HF: alta frecuencia. LF: baja frecuencia. Relación HF/LF: relación entre alta y baja frecuencia.

DE: desviación estándar.

$\mathrm{P}=$ valor $\mathrm{p}$ de la prueba de $t$-student .

El tratamiento en el grupo total no produjo diferencias significativas en alguno de los parámetros de dominio de tiempo o de frecuencia de la variabilidad de la frecuencia cardiaca, Cuadro 4. 
Grupo A. El efecto de tratamiento con moxibustión en los parámetros de la variabilidad de la frecuencia cardiaca en el grupo A con predominio del tono parasimpático se muestra en Cuadro 5.

Cuadro 5. Efecto del tratamiento con moxibustión en los parámetros de dominio de tiempo y frecuencia en el grupo A con predominio parasimpático.

\begin{tabular}{|c|c|c|c|}
\hline & $\begin{array}{c}\text { Basal } \\
\text { (media } \pm \text { DE) }\end{array}$ & $\begin{array}{c}\text { Tratado } \\
\text { (media } \pm \mathrm{DE})\end{array}$ & $\mathbf{P}$ \\
\hline \multicolumn{4}{|c|}{ Dominio de tiempo } \\
\hline PNN50 & $33.7 \pm 5.8$ & $34.8 \pm 7.5$ & 0.4436 \\
\hline RMSSD & $61.1 \pm 3.7$ & $60.9 \pm 5.4$ & 0.8189 \\
\hline \multicolumn{4}{|c|}{ Dominio de Frecuencia } \\
\hline $\mathrm{HF}$ & $43.5 \pm 21.8$ & $35.8 \pm 17.1$ & 0.1293 \\
\hline $\mathrm{LF}$ & $56.5 \pm 21.8$ & $64.2 \pm 17.1$ & 0.1293 \\
\hline LF/HF & $1.8 \pm 1.2$ & $2.5 \pm 1.8$ & 0.3215 \\
\hline \multicolumn{4}{|c|}{$\begin{array}{l}\text { PNN50. Número de intervalos adyacentes que varían por más de } 50 \mathrm{~ms} \\
\text { expresado en porcentaje. RSSMD. Cuadrado de la raíz media de la unión de } \\
\text { los intervalos R-R adyacentes. }\end{array}$} \\
\hline \multicolumn{4}{|c|}{$\begin{array}{l}\text { HF: alta frecuencia. LF: baja frecuencia. Relación HF/LF: relación entre alta y } \\
\text { baja frecuencia. }\end{array}$} \\
\hline \multicolumn{4}{|c|}{ DE: desviación estándar. } \\
\hline \multicolumn{4}{|c|}{$\mathrm{P}=$ valor $\mathrm{p}$ de la prueba de $t$-student } \\
\hline
\end{tabular}

El tratamiento en el grupo A no produjo diferencias significativas en alguna de los parámetros de dominio de tiempo o de frecuencia de la variabilidad de la frecuencia cardiaca, Cuadro 5. 
Grupo B. El efecto de tratamiento con moxibustión en los parámetros de la variabilidad de la frecuencia cardiaca en el grupo B con predominio del tono simpático se muestra en el Cuadro 6.

El tratamiento en el grupo B no produjo diferencias significativas en alguna de los parámetros de dominio de frecuencia o de tiempo de la VFC, ver Cuadro 6.

El tratamiento con moxibustión en V15 mostró efectos diferenciados de direccionalidad contraria en las variables de dominio de tiempo y frecuencia en los grupos A y B de predominio parasimpático y simpático, de manera respectiva. Las mayores diferencias se observaron en HF y LF, ver Cuadro 7. 
Cuadro 6. Efecto del tratamiento con moxibustión en los parámetros de dominio de tiempo y frecuencia de la variabilidad de la frecuencia cardiaca en el grupo B con predominio simpático.

\begin{tabular}{ccc}
\hline Basal & Tratado & $\mathbf{P}$ \\
$($ media $\pm \mathrm{DE})$ & $($ media $\pm \mathrm{DE})$ & \\
\hline
\end{tabular}

\section{Dominio de tiempo}

\begin{tabular}{lccc}
\hline PNN50 & $26.9 \pm 3.7$ & $25.6 \pm 1$ & 0.6142 \\
\hline RMSSD & $52.1 \pm 7.9$ & $52.5 \pm 14$ & 0.8952 \\
\hline
\end{tabular}

Dominio de frecuencia

\begin{tabular}{lccc}
\hline HF & $20.7 \pm 3.2$ & $23.2 \pm 2.6$ & 0.2487 \\
\hline LF & $79.3 \pm 3.2$ & $76.8 \pm 2.6$ & 0.2487 \\
\hline LF/HF & $4 \pm 1$ & $3.4 \pm 0.6$ & 0.2974
\end{tabular}

PNN50: número de intervalos adyacentes que varían por más de $50 \mathrm{~ms}$ expresado en porcentaje. RSSMD: cuadrado de la raíz media de la unión de los intervalos R-R adyacentes.

HF: alta frecuencia. LF: baja frecuencia. Relación HF/LF: relación entre alta y baja frecuencia.

DE: desviación estándar.

$\mathrm{P}=$ valor $\mathrm{p}$ de la prueba de $t$-student . 
Comparación del efecto del tratamiento en V15 en la VFC en el grupo A versus el grupo B

Cuadro 7. Comparación del efecto del tratamiento de V15 con moxibustión en el grupo A con predominio de tono parasimpático versus el grupo B con predominio de tono simpático.

$\begin{array}{lll}\text { Delta grupo } A & \text { Delta grupo } B & p \\ (\text { media } \pm D E) & (\text { media } \pm D E) & \end{array}$

Dominio de tiempo

\begin{tabular}{lccc}
\hline PNN50 & $1.1 \pm 3.6$ & $-1.3 \pm 6.7$ & 0.4086 \\
\hline RMSSD & $-0.2 \pm 2.4$ & $0.4 \pm 8$ & 0.8450 \\
\hline
\end{tabular}

Dominio de frecuencia

\begin{tabular}{lccc}
\hline $\mathrm{HF}$ & $-7.7 \pm 11.7$ & $2.4 \pm 5$ & 0.0557 \\
\hline $\mathrm{LF}$ & $7.7 \pm 11.7$ & $-2.4 \pm 5$ & 0.05566 \\
\hline $\mathrm{LF} / \mathrm{HF}$ & $0.7 \pm 1.6$ & $-0.6 \pm 1.3$ & 0.1448 \\
\hline
\end{tabular}

Delta: valor del tratado - valor del basal; para cada uno de los participantes.

PNN50: número de intervalos adyacentes que varían por más de $50 \mathrm{~ms}$ expresado en porcentaje.

RSSMD: cuadrado de la raíz media de la unión de los intervalos R-R adyacentes.

HF: alta frecuencia. LF: baja frecuencia. Relación HF/LF: relación entre alta y baja frecuencia.

DE: desviación estándar.

$\mathrm{P}=$ valor $\mathrm{p}$ de la prueba de $t$-student.

Comparaciones porcentuales de los cambios originados por el tratamiento en los parámetros de dominio de tiempo y frecuencia de la variabilidad de la frecuencia cardiaca en el grupo A versus grupo B 
Dominio de tiempo. Las diferencias porcentuales en los parámetros de dominio de tiempo consecutivos al tratamiento se muestran de las Figuras 3 y 4.

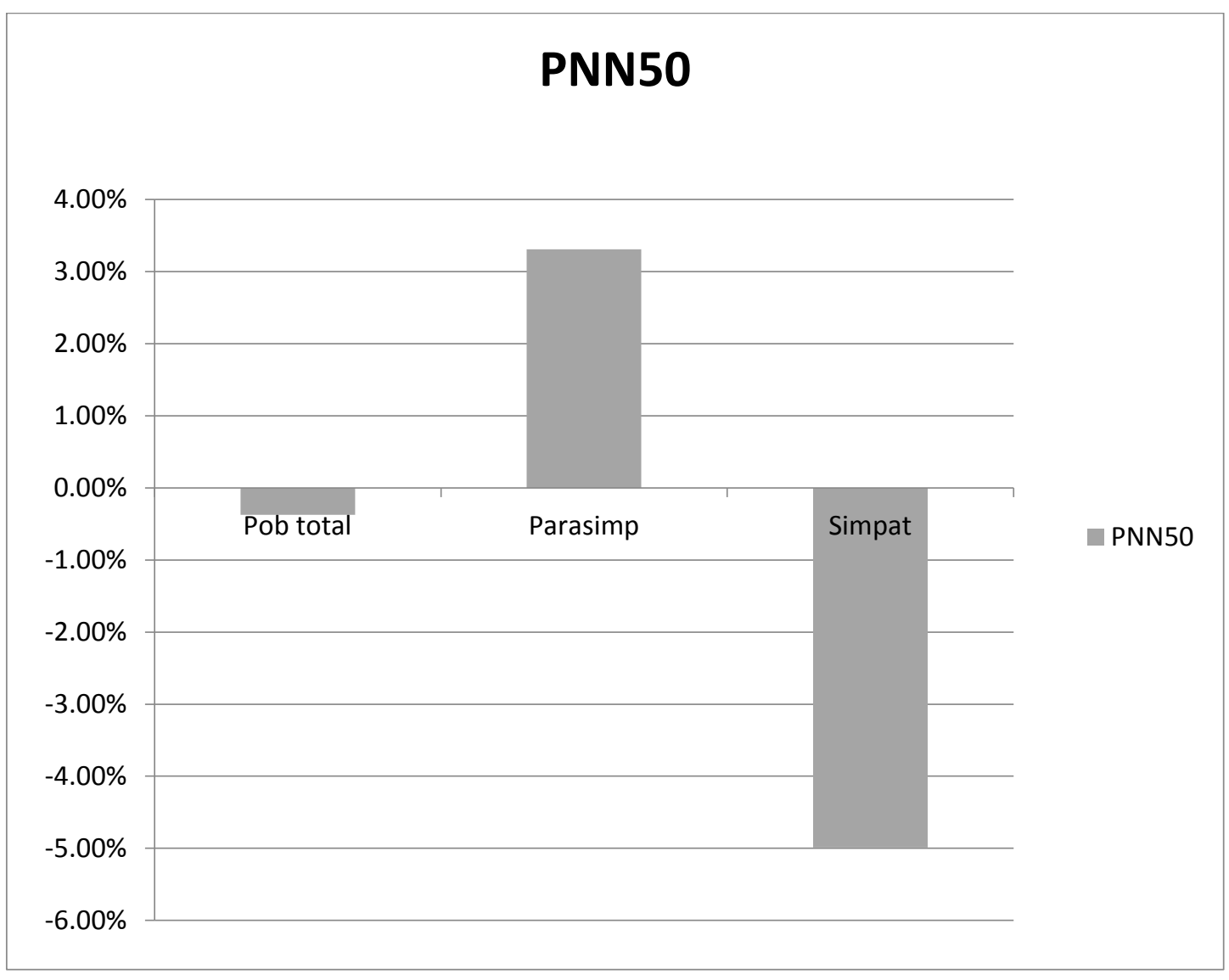

Figura 3. Cambios porcentuales del parámetro de tiempo PNN50 de la variabilidad de la frecuencia cardiaca facilitados por el tratamiento en el punto V15.

En la Figura 3 que los cambios secundarios al tratamiento con moxibustión en el valor de dominio de tiempo PNN50 fueron menores y de carácter opuesto en los grupos A y B. 


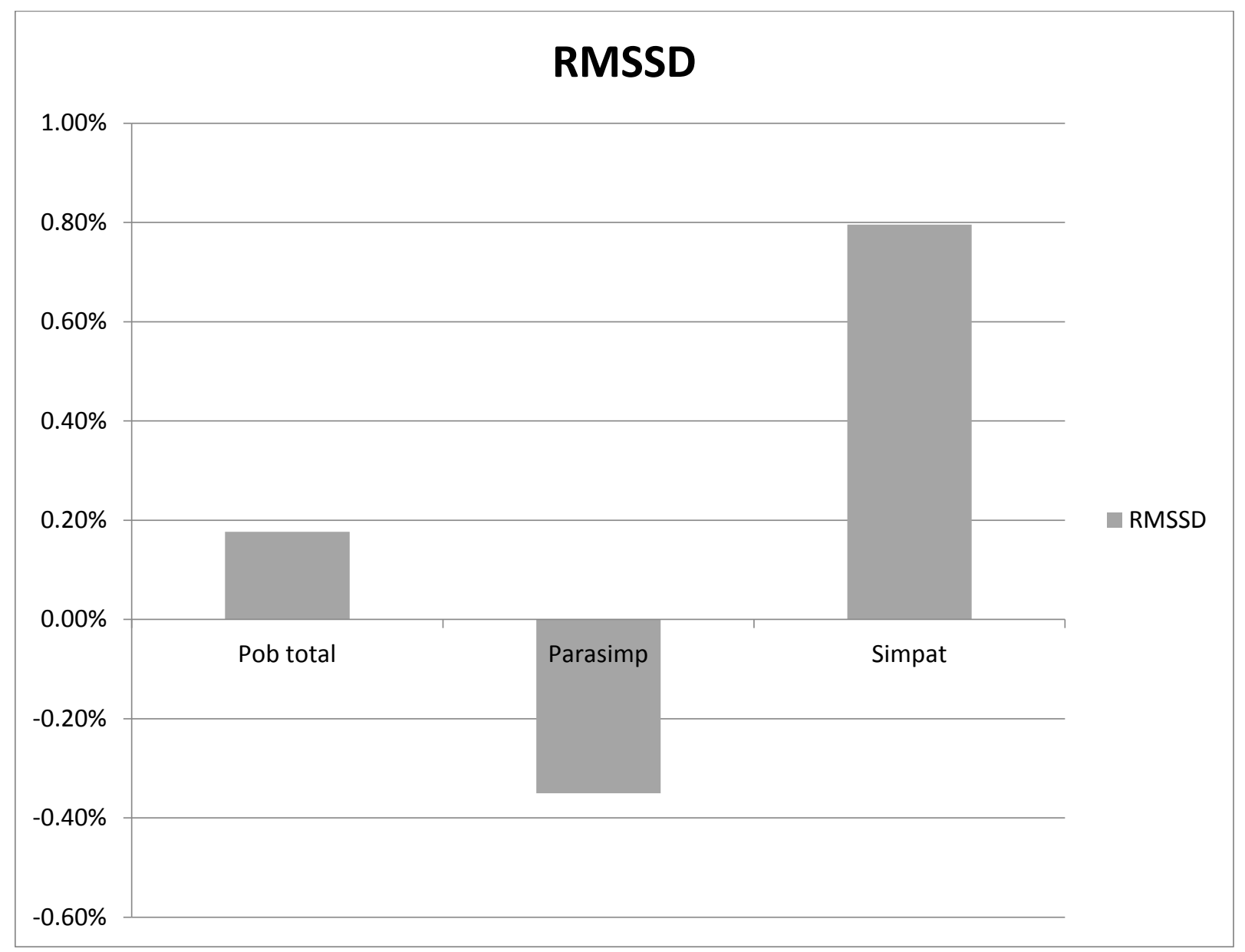

Figura 4. Cambios porcentuales en los tres grupos estudiados del valor de dominio de tiempo RMSSD de la variabilidad de la frecuencia cardiaca facilitados por el tratamiento en el punto V15.

En el caso de los valores de dominio de tiempo (PNN50 y RMSSD) los cambios relacionados con el tratamiento con moxibustión en V15 fueron no significativos. 
Dominio de frecuencia. Las diferencias porcentuales en los parámetros de dominio de frecuencia consecutivos al tratamiento se muestran de la Figuras 5, 6 y 7.

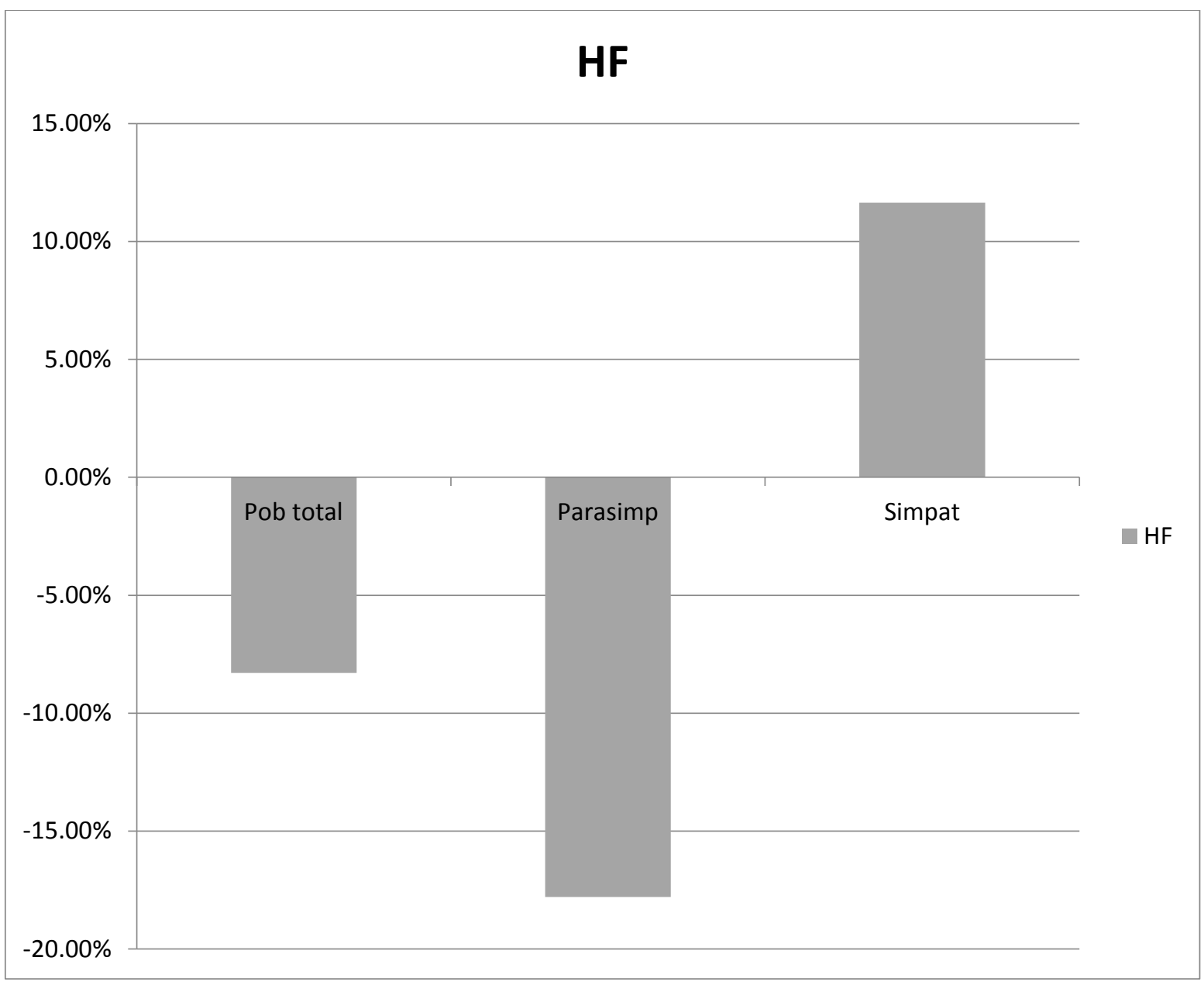

Figura 5. Cambios porcentuales en los tres grupos estudiados del valor de dominio de frecuencia HF de la variabilidad de la frecuencia cardiaca facilitados por el tratamiento en el punto V15. 


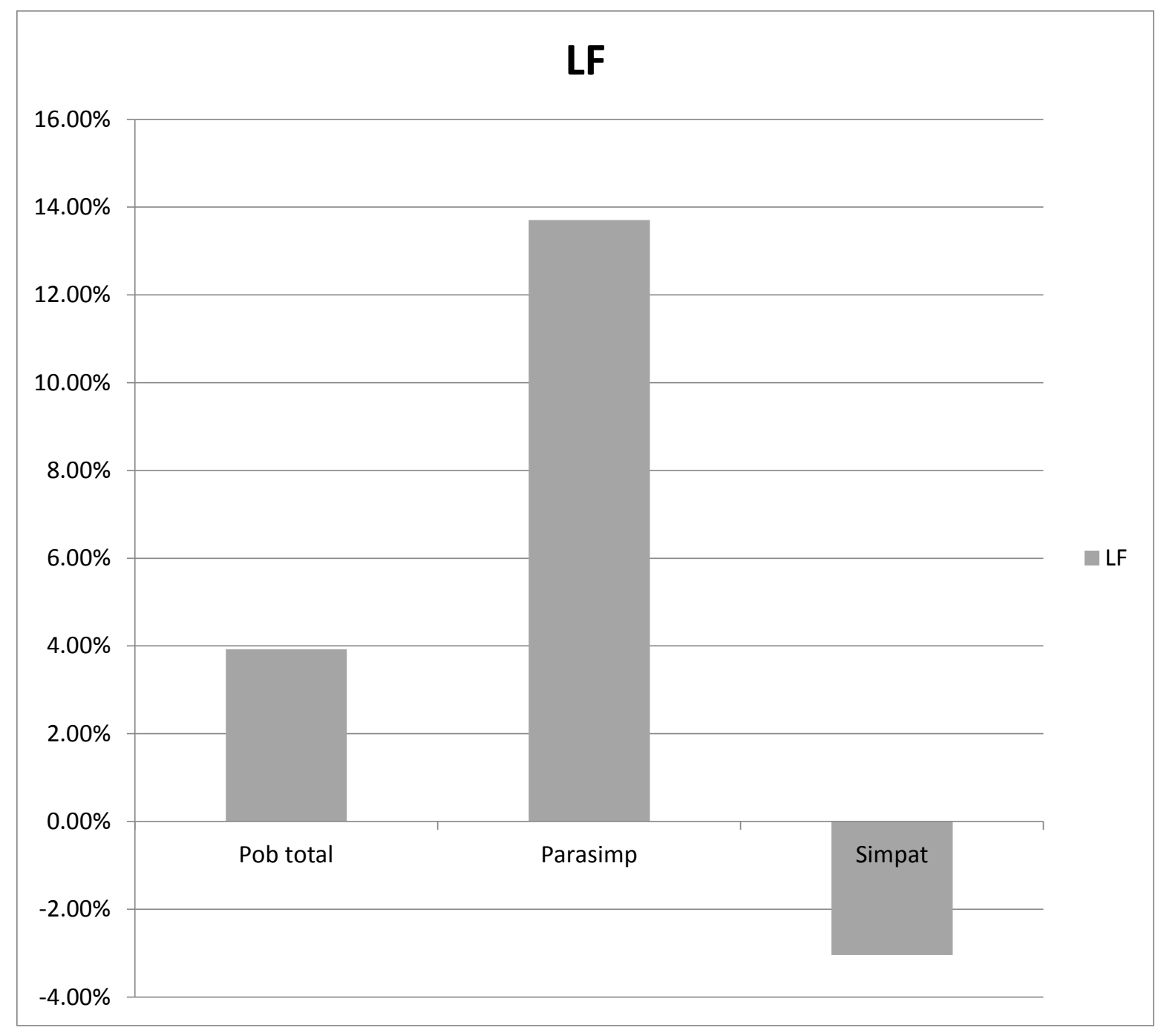

Figura 6. Cambios porcentuales en los tres grupos estudiados del valor de dominio de frecuencia LF de la variabilidad de la frecuencia cardiaca facilitados por el tratamiento en el punto V15.

En el caso de los valores de dominio de frecuencia LF y LF/HF los cambios secundarios al tratamiento con moxibustión en V15 fueron más acentuados y también de sentido contrario en los grupos A y B. 


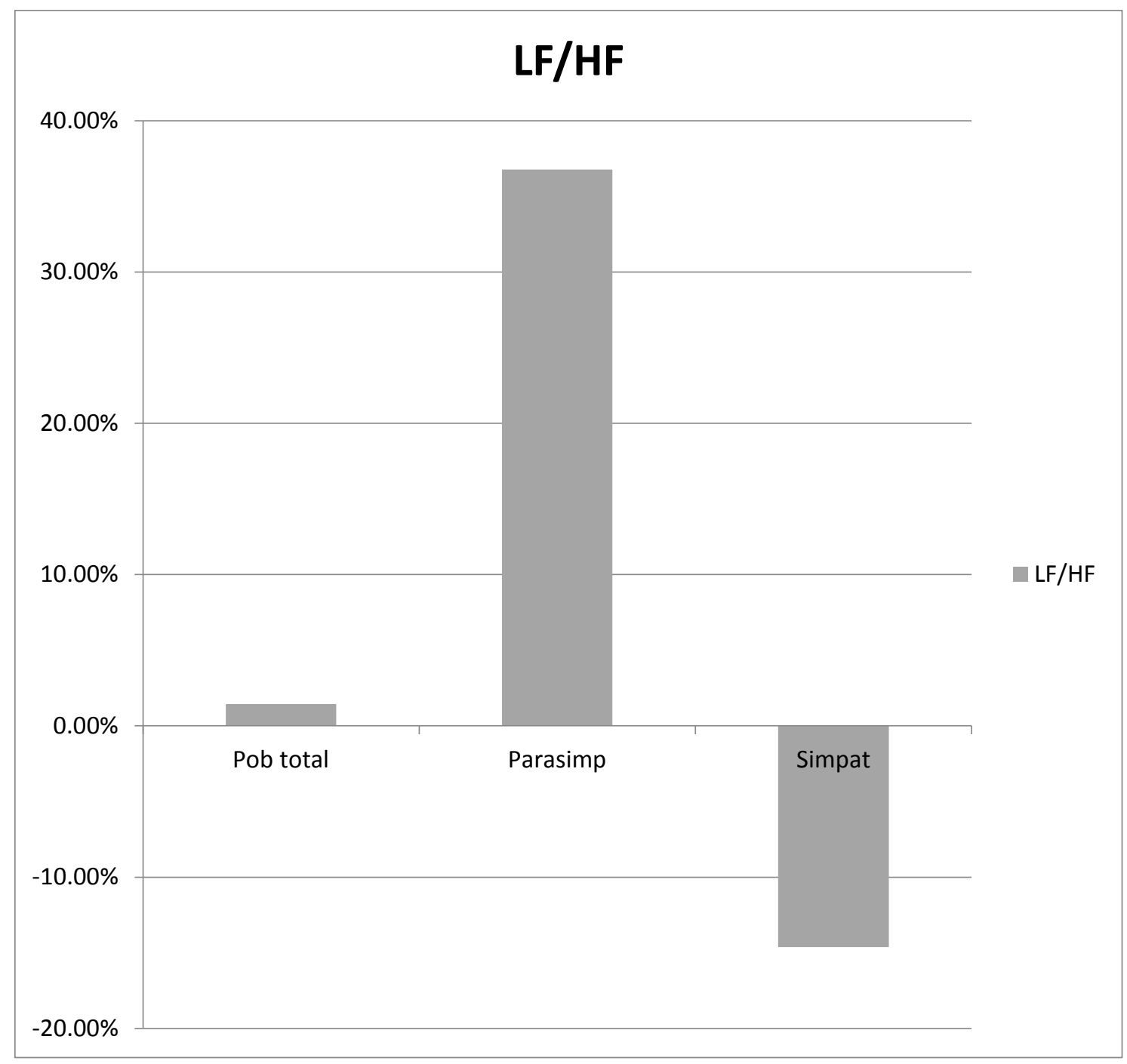

Figura 7. Cambios porcentuales en los tres grupos estudiados del valor de dominio de frecuencia LF/HF de la variabilidad de la frecuencia cardiaca facilitados por el tratamiento en el punto V15.

Respecto a los valores de dominio de frecuencia (HF, LF y LF/HF) los cambios relacionados con el tratamiento con moxibustión en V15 fueron de proporciones mucho mayores a los de los valores de dominio de tiempo. 
Cuadro 8. Comparación del efecto del tratamiento con moxibustión en el grupo A con predominio de tono parasimpático versus el grupo B con predominio de tono simpático.

\begin{tabular}{lccc}
\hline & $\begin{array}{c}\text { Delta grupo A } \\
(\text { media } \pm \mathrm{DE})\end{array}$ & $\begin{array}{c}\text { Delta grupo B } \\
(\text { media } \pm \mathrm{DE})\end{array}$ & $\mathrm{P}$ \\
\hline Dominio de tiempo & & & \\
\hline PNN50 & $1.1 \pm 3.6$ & $-1.3 \pm 6.7$ & 0.4086 \\
\hline RMSSD & $-0.2 \pm 2.4$ & $0.4 \pm 8.0$ & 0.8450 \\
\hline & & & \\
\hline Dominio de frecuencia & & & 0.0556 \\
\hline HF & $-7.7 \pm 11.7$ & $2.4 \pm 5.0$ & 0.0556 \\
\hline LF & $7.7 \pm 11.7$ & $-2.4 \pm 5.0$ & 0.1448 \\
\hline LF/HF & $0.7 \pm 1.6$ & $-0.6 \pm 1.3$ &
\end{tabular}

Delta: valor del tratado - valor del basal; para cada uno de los participantes; expresados como media \pm desviación estándar.

PNN50: número de intervalos adyacentes que varían por más de $50 \mathrm{~ms}$ expresado en porcentaje.

RSSMD: cuadrado de la raíz media de la unión de los intervalos $R-R$ adyacentes.

HF: alta frecuencia. LF: baja frecuencia. Relación HF/LF: relación entre alta y baja frecuencia.

DE: desviación estándar.

$\mathrm{P}=$ valor $\mathrm{p}$ de la prueba de $\mathrm{t}-\mathrm{student}$ pareada.

${ }^{*} p<0.05$ 
Correlaciones de Pearson entre los parámetros de dominio de tiempo y de frecuencia en los grupos estudiados

Los coeficientes de correlación de Pearson así como su significación estadística entre los diferentes parámetros de dominio de tiempo y frecuencia para la muestra total y grupos A y B se muestran en los Cuadros 9, 10 y 11 de manera respectiva.

Cuadro 9. Coeficientes de correlación de Pearson entre los parámetros de variabilidad de la frecuencia cardiaca en la población total posteriores al tratamiento con moxibustión en V15.

\begin{tabular}{lcccc}
\hline & RMSSD & HF & LF & LF/HF \\
\hline PNN50 & $r=0.7242$ & $r=-0.3000$ & $r=0.3000$ & $r=0.3645$ \\
& $p=0.0017$ & $p=0.1487$ & $p=0.1487$ & $p=0.1000$ \\
\hline RMSSD & & $r=-0.0452$ & $r=0.0452$ & $r=0.07268$ \\
& & $p=0.4390$ & $p=0.4390$ & $p=0.4024$ \\
\hline HF & & $r=-1$ & $r=-0.8233$ \\
& & & $p<0.0001$ & $p=0.0001$ \\
\hline LF & & & $r=0.8233$ \\
& & & & $p=0.0001$ \\
\hline
\end{tabular}

PNN50: número de intervalos adyacentes que varían por más de $50 \mathrm{~ms}$ expresado en porcentaje. RSSMD: es el cuadrado de la raíz media de la unión de los intervalos $\mathrm{R}-\mathrm{R}$ adyacentes.

HF: alta frecuencia. LF: baja frecuencia. Relación HF/LF: relación entre alta y baja frecuencia.

r: coeficiente de correlación de Pearson; p: valor estadístico de la correlación de Pearson.

Cuadro 10. Coeficiente de correlación de Pearson de los parámetros de variabilidad de la frecuencia cardiaca modificada por la moxibustión en V15 en los sujetos del grupo A con predominancia de tono parasimpático. 


\begin{tabular}{lcccc}
\hline & RMSSD & HF & LF & LF/HF \\
\hline PNN50 & $r=-0.0183$ & $r=-0.0216$ & $r=0.02165$ & $r=-0.0650$ \\
& $\mathrm{p}=0.4844$ & $\mathrm{p}=0.4816$ & $\mathrm{p}=0.4816$ & $\mathrm{p}=0.4449$ \\
\hline RMSSD & & $\mathrm{r}=0.2552$ & $\mathrm{r}=-0.2552$ & $\mathrm{r}=-0.777$ \\
& & $\mathrm{p}=0.2903$ & $\mathrm{p}=0.2905$ & $\mathrm{p}=0.0199^{*}$ \\
\hline HF & & $\mathrm{r}=-1$ & $\mathrm{r}=-0.7548$ \\
& & & $\mathrm{p}=0.0001^{*}$ & $\mathrm{p}=0.0249^{*}$ \\
\hline LF & & & $\mathrm{r}=0.7548$ \\
& & & $\mathrm{p}=0.0249^{*}$ \\
\hline
\end{tabular}

PNN50: número de intervalos adyacentes que varían por más de $50 \mathrm{~ms}$ expresado en porcentaje.

RSSMD: es el cuadrado de la raíz media de la unión de los intervalos R-R adyacentes.

HF: alta frecuencia. LF: baja frecuencia. Relación HF/LF: relación entre alta y baja frecuencia.

r: coeficiente de correlación de Pearson; p: valor estadístico de la correlación de Pearson.

${ }^{*} p<0.05$ 
Cuadro 11. Coeficientes de correlación de Pearson de los parámetros de variabilidad de la frecuencia cardiaca modificada por la moxibustión en V15 en los sujetos del grupo B con predominancia de tono simpático.

\begin{tabular}{lllll}
\hline & RMSSD & HF & LF & LF/HF \\
\hline PNN50 & $r=0.9049$ & $r=-0.5807$ & $r=0.5807$ & $r=0.5771$ \\
& $p=0.0025^{*}$ & $p=0.0858$ & $p=0.0858$ & $p=0.0874$ \\
\hline RMSSD & & $r=-0.4106$ & $r=0.4106$ & $r=0.4516$ \\
& & $p=0.1800$ & $p=0.1800$ & $p=0.1545$ \\
\hline HF & & $r=-1$ & $r=-0.9894$ \\
& & & $p<0.0001^{*}$ & $p<0.0001^{*}$ \\
\hline LF & & & $r=0.9894$ \\
& & & & $p<0.0001^{*}$ \\
\hline
\end{tabular}

PNN50: número de intervalos adyacentes que varían por más de $50 \mathrm{~ms}$ expresado en porcentaje.

RSSMD: es el cuadrado de la raíz media de la unión de los intervalos RR adyacentes.

HF: alta frecuencia. LF: baja frecuencia. Relación HF/LF: relación entre alta y baja frecuencia.

r: coeficiente de correlación de Pearson; p: valor estadístico de la correlación de Pearson.

${ }^{*} p<0.05$

Los coeficientes de correlación de Pearson entre las variables de tiempo y frecuencia de la VFC solo mostraron valores significativos entre las variables de frecuencia entre los tres grupos estudiados. 


\section{DISCUSIÓN}

\section{Sumario de resultados}

La comparación basal de los parámetros de dominio y frecuencia de la variabilidad de la frecuencia cardiaca del grupo A versus grupo B mostró diferencias significativas en todos los valores del dominio de tiempo y frecuencia de la VFC.

El tratamiento con moxibustión en V15 no originó cambios significativos en los valores de los dominios de tiempo y frecuencia de la VFC de la muestra total. En el grupo A se observaron cambios constantes pero no significativos en los valores del dominio de frecuencia de la VFC con disminución de HF e incremento de LF y LF/HF. En el grupo $B$ no se observaron cambios significativos en los valores de la VFC. Sin embargo, los cambios originados por el tratamiento en los parámetros de tiempo y frecuencia de la VFC de los grupos A versus $B$ tuvieron direccionalidad contraria. En el grupo A de predominio de tono parasimpático los cambios fueron en sentido de disminuir este predominio. Por el contrario, en el grupo B de predominio de tono simpático los cambios fueron en el sentido de disminuir esa orientación. Las mayores diferencias entre los grupos A y B se observaron en HF y LF. El análisis de Pearson entre las variables de tiempo y frecuencia de la VFC solo mostraron correlaciones significativas desde el punto de vista estadístico entre los valores de frecuencia en los tres grupos estudiados. 
Las enfermedades cardiovasculares son la principal causa de morbilidad y mortalidad en los países en desarrollo. La incidencia va en aumento y contribuye a la gran carga de salud de un país. La mayoría de los factores de riesgo para enfermedades cardiovasculares pueden modificarse. La VFC es una herramienta importante para evaluar la influencia del sistema nervioso autónomo en el sistema cardiovascular y valorar las consecuencias de los factores ambientales que contribuyen al riesgo cardiovascular. La VFC puede servir como un método sensible de evaluación de los cambios tempranos en la función del SNA.

En este estudio, se examinó el efecto a corto plazo de la aplicación de la estimulación de V15 en la función del SNA evaluada por medio de la VFC. Los marcadores del dominio del tiempo como PNN50 y RMSSD se modificaron en forma constante y en algunos casos de manera significativa después del tratamiento con moxibustión. Estos cambios en el equilibrio parasimpáticosimpático del SNA fueron diferentes en los grupos A y B con predominio de los tonos parasimpático o simpático, de manera respectiva. El incremento o disminución de PNN50 y RMSSD indican un aumento o disminución del tono parasimpático, de manera respectiva; que se relaciona a su vez con una variación en la duración del ciclo cardíaco.

Los resultados obtenidos indican que la moxibustión en el punto V15 en sujetos sanos provoca modificaciones constantes en los componentes de dominio de frecuencia; no así en el dominio de tiempo, en los que los cambios son ínfimos. 
Respecto a los índices del dominio de frecuencia de la VFC, el índice HF se acepta que se relaciona con el nivel del tono parasimpático. El espectro HF es la potencia en cada uno de los segmentos de 5 minutos en el rango de 0.15 a 0.4 Hz. Esta banda refleja la actividad parasimpática o vagal y con frecuencia se denomina banda respiratoria porque corresponde a las variaciones de la frecuencia cardíaca relacionadas con el ciclo respiratorio. Estos cambios en la frecuencia cardíaca se conocen como arritmia sinusal respiratoria (ASR). De esta manera, se acepta en genera que el poder de la HF refleja la arritmia sinusal respiratoria, que está mediada por el sistema colinérgico parasimpático (Fatisson y Cols. 2016).

Mientras que la modificación de la magnitud de LF puede ser atribuida a cambios en el grado de inhibición del área posterior o simpática del hipotálamo que optimiza las respuestas simpáticas del cuerpo al estrés (Billman 2013).

Se ha informado de evidencia de que la potencia de LF podría estar relacionada con la modulación barorreflexa relacionada con impulsos autónomos cardiacos. Además se ha demostrado que la estimulación del seno carotídeo incrementa la potencia de LF en individuos con función barorrefleja normal. La potencia LF evaluada durante el reposo en posición sentada, de pie o durante la maniobra de Valsalva parece proporcionar un índice no de tono cardiaco simpático si no de función barorrefleja (Moak y Cols. 2009; Rahman y Cols. 2011; Shaffer 2004). 
Por otra parte, se dice de manera común que las modificaciones de la relación LF/HF son un marcador del equilibrio del SNA. Se considera que una relación LF/HF superior a 4.8 refleja el predominio simpático y aquella $<1.3$ una modulación vagal predominante. Sin embargo, el concepto de que la relación LF/HF indica el estado de equilibrio parasimpático/simpático es cada vez menos aceptado (Goldstein y Cols. 2011).

Mecanismos de V15. Se ha demostrado en modelos experimentales en animales mediante técnicas de inmunofluorescencia que existen conexiones nerviosas periféricas entre los puntos de V 15 (Xinshu), PC 6 (Neiguan) y el corazón. Los resultados muestraron que el corazón y el punto V15 se conectan de manera directa entre sí mediante proyecciones divergentes de las neuronas sensoriales primarias y puede haber una conexión directa o indirecta entre las neuronas posganglionares simpáticas de inervación del corazón y el punto V15, además de conexiones a través del sistema nervioso central. Los autores proponen que sus resultados proporcionan una evidencia de los mecanismos de correlación entre los puntos de acupuntura y los órganos tóraco abdominales (Shulin y Yuliang, 1996).

Otra relación anatomo-neurológica que puede estar involucrado en este proceso, es la estimulación del núcleo del tracto solitario, el cual recibe aferencias sensoriales del nervio vago y provee señales eferentes al hipocampo para 
regular la actividad cardiovascular; y algunas de sus neuronas están correlacionadas con índices hemodinámicos (Wang y Cols. 2018)

Se sabe que el núcleo del tracto solitario procesa de manera diferenciada la actividad simpática y parasimpática, posiblemente controlando estas vías de manera independiente, lo que explicaría que la frecuencia cardiaca pueda ser modulada por estímulos con acupuntura (Tjen-A-Looi y Cols 2014).

Así también otros informes han indicado la relación del estímulo de puntos de acupuntura con la medula rostral ventrolateral, que es un centro cerebral que envía axones externos a la medula espinal para regular funciones cardiacas (Lim y Cols. 2016). Y que es responsable de la actividad simpática asociada con la función cardiovascular. El cual envía proyecciones a las neuronas simpáticas preganglionares en el núcleo intermedio lateral de la medula espinal vía el tracto retículo espinal.

Por otra parte, los resultados del estudio presente contradicen uno de los dogmas de la medicina tradicional China, que según la manera en que se manipula la moxa, se estimula (tonifica) o relaja al organismo (dispersa). De esta manera, se pudo comprobar que aplicando la moxa sin algún tipo de manejo especial se modifica el equilibrio parasimpático/simpático, en forma solo dependiente del estado basal de equilibrio del SNA en el sujeto. 
Un estudio previo (Hsu y Cols. 2006) mostró que la electroestimulación de V15 produjo incremento de HF y disminución de LF en voluntarios sanos. En otro estudio donde se compararon diferentes tipos de estimulación en V15 en la VFC se encontró que acupuntura manual y aplicación de campos electromagnéticos indujeron aumento de HF y disminución de LF equivalentes a un incremento de actividad parasimpática; mientras que estimulación láser indujo un efecto contrario con disminución de HF y aumento de LF/HF equivalentes a incremento de actividad simpática (Lee y Cols. 2016).

Se estudiaron las conexiones nerviosas periféricas entre los puntos de V15 (Xinshu), PC6 (Neiguan) y el corazón en el conejo mediante técnicas de fluorescencia. Los resultados muestraron que el corazón y el punto V15 se conectan directamente entre sí mediante proyecciones divergentes de las neuronas sensitivas primarias y puede haber una conexión directa o indirecta entre las neuronas posganglionares simpáticas de inervación del corazón y el punto PC6, además de conexiones a través del sistema nervioso central sistema. Si se toma solamente las poblaciones totales de los estudios, los resultados serán contradictorios a otras investigaciones.

Por ejemplo en el de Hsu y Cols. (2006), se encontró que la aplicación de electroacupuntura en V15 incrementó la actividad parasimpático y en la investigación presente se observó un incremento de la actividad simpática. 
Respecto a la moxibustión se propone que la moxibustión ejerce su efecto sobre los puntos de acupuntura, no solo a través de energía calorífica, también a través de resonancia infrarroja y la liberación de sustancias activas a partir de la planta Artemisia sp (Yang y Cols. 2012). 


\section{LIMITACIONES DEL ESTUDIO}

Las limitaciones potenciales de esta investigación son las siguientes: que se realizó una estimulación moderada con moxibustión del punto V15; sin embargo, se lograron cambios constantes y en algunos casos significativos en las variables estudiadas; debido al número reducido de sujetos, no se pudo realizar un análisis multivariado, por ejemplo respecto al sexo, la edad o la IMC de los sujetos; y respecto a la variabilidad de la frecuencia cardiaca, una limitación del estudio es que ésta fue calculada con un periodo de intervalos $R R$ de un periodo inmediato a la estimulación con moxibustión.

Debido a los daños del Edificio $S$ de la UAM Iztapalapa y la pérdida del Laboratorio de Acupuntura y Enfermedades Crónicas y Degenerativas no se pudo contar con un área acondicionada en forma especial para este tipo de estudio. 


\section{PERSPECTIVAS}

Debido a que la modificación de la VFC es mediada en parte por mecanismos relacionados con la actividad del sistema nervioso autónomo en cualquiera de sus dos vertientes parasimpática o simpática, se pueden proponer estudios en los que se apliquen agonistas antagonistas colinérgicos o adrenérgicos para observar si se modifica la acción del punto de acupuntura examinado.

Otra línea de investigación potencial es analizar si otros estímulos diferentes a la moxibustión como la acupuntura o la aplicación de láser en el punto V15 son capaces de modificar las variables de los dominios de tiempo y frecuencia de la variabilidad de la frecuencia cardiaca.

Dado que en este estudio solo se utilizó el punto V15 del lado izquierdo, en futuras investigaciones se puede estudiar si la aplicación del punto del lado derecho da resultados iguales; incluso si usando el punto de manera bilateral se incrementa la magnitud de la respuesta. 


\section{CONCLUSIONES}

La estimulación del punto V15 mediante moxibustión modificó la VFC en forma diferenciada en los grupos estudiados dependiendo del estado basal del equilibrio parasimpático/simpático. En los sujetos con predominio basal parasimpático el tratamiento con moxa tendió a disminuir este predominio; mientras que en los sujetos con predominio basal simpático, se presentó un aumento de la actividad parasimpática.

El tratamiento con moxibustión en V15 modificó de manera más marcada los valores del dominio de frecuencia que los de tiempo. Consideramos que en futuros estudios en que se intente modificar la VFC con estimulación de puntos de acupuntura deberá considerarse el estado basal del equilibrio parasimpático/simpático. 


\section{REFERENCIAS BIBLIOGRÁFICAS}

1. Bansilal S, Castellano JM, Fuster V (2015) Global burden of CVD: focus on secondary prevention of cardiovascular disease. Int J Cardiol. 201 Suppl 1:S1-7.

2. Benichou T, Pereira B, Mermillod M, Tauveron I, Pfabigan D, Maqdasy S, Dutheil F (2018) Heart rate variability in type 2 diabetes mellitus: A systematic review and meta-analysis. PLoS One; 13(4): e0195166.

3. Billman GE (2013) The LF/HF ratio does not accurately measure cardiac sympatho-vagal balance. Front Physiol. 4: 26.

4. Draghici AE, Taylor JA (2016) The physiological basis and measurement of heart rate variability in humans. J Physiol Anthropol. 35(1):22.

5. Fatisson J, Oswald V, Lalonde F (2016) Influence diagram of physiological and environmental factors affecting heart rate variability: an extended literature overview. Heart Int. 11(1): e32-e40.

6. Gallo J, Farbiarz J, Álvarez D (1999) Análisis espectral de la variabilidad de la frecuencia cardíaca. Atreia/Vol $12 / \mathrm{N}^{\circ} 2$.

7. Goldstein DS, Bentho O, Park MY, Sharabi Y (2011) Low-frequency power of heart rate variability is not a measure of cardiac sympathetic tone but may be a measure of modulation of cardiac autonomic outflows by baroreflexes. Exp Physiol. 96(12):1255-61. 
8. Gutiérrez JP, Rivera- J, Shamah T, Villalpando S, Franco A, Cuevas L, et al. (2012) Encuesta Nacional de Salud y Nutrición 2012. Resultados Nacionales. Cuernavaca: Instituto Nacional de Salud Pública.

9. Jun Z (1984) Fundamentos de Acupuntura y moxibustion de China. Ediciones en Lenguas Extranjeras. Beijing, República Popular China.

10. Lees T, Shad-Kaneez F, Simpson AM, Nassif NT, Lin Y, Lal S (2018) Heart Rate Variability as a Biomarker for Predicting Stroke, Post-stroke Complications and Functionality. Biomark Insights; 13: 1177271918786931.

11. Lim HD, Kim MH, Lee CY, Namgung U (2016) Anti-Inflammatory Effects of Acupuncture Stimulation via the Vagus Nerve. PLoS One 11(3):e0151882.

12. Maoliang Q (1993), Chinese Acupuncture and Moxibustion. Churchill Livingstone, London.

13. Moak JP, Goldstein DS, Eldadah BA, Saleem A, Holmes C, Pechnik S, Sharabi $Y$ (2009). Supine low-frequency power of heart rate variability reflects baroreflex function, not cardiac sympathetic innervation. Cleve Clin J Med. Apr;76 Suppl 2:S51-9.

14. Rahman F, Pechnik S, Gross D, Sewell LT, Goldstein DS (2011). LF Power Reflects Baroreflex Function, Not Cardiac Sympathetic Innervation. Clin Auton Res; 21(3): 133-141. 
15. Rajendra Acharya U, Paul Joseph K, Kannathal N, Lim CM, Suri JS (2006) Heart rate variability: a review. Med Biol Eng Comput. 44: 10311051.

16. Seely AJ, Macklem PT (2004) Complex systems and the technology of variability analysis. Crit Care; 8(6): R367-84.

17. Noh SH, Lee BR, Yim YK (2014) Single and triple moxibustion with large, indirect moxa induced differential effects on skin temperature and blood perfusion in healthy human subjects: Counterevidence to a previous report Original Research Article. Complementary Therapies in Medicine. 22 (2):311-319

18. Shaffer F, McCraty R, Zerr CL (2014) A healthy heart is not a metronome: an integrative review of the heart's anatomy and heart rate variability. Front Psychol. 5: 1040.

19. Shulin C, Yuliang L (1996) Nervous basis of correlation between Neiguan (PC6), Xinshu (BL15) Points and the Heart. Chin Acup \& Moxibustion; 12: sin pp.

20. Syuu Y, Matsubara H, Kiyooka T, Hosogi S, Mohri S, Araki J, Ohe T, Suga $H$ (2001) Cardiovascular beneficial effects of electroacupuncture at Neiguan (PC-6) acupoint in anesthetized open-chest dog. Jpn $\mathrm{J}$ Physiol. 51(2):231-8.

21. Task Force of the European Society of Cardiology and the North American Society of Pacing and Electrophysiology (1996). Heart rate 
variability: standards of measurement, physiological interpretation and clinical use. Circulation; 93(5): 1043-1065.

22. Tjen-A-Looi SC, Guo ZL, Longhurst JC (2014) GABA in nucleus tractus solitarius participates in electroacupuncture modulation of cardiopulmonary bradycardia reflex. Am J Physiol Regul Integr Comp Physiol. 2014 Dec 1;307(11):R1313-23.

23. Tsuji H, Venditti Jr FI, Manders ES (1994) Reduced heart rate variability and mortality risk in an elderly cohort: the Framingham Heart Study. Circulation 90 (2): 878-883.

24. Wang K, Wu SB, Cui S, Xiang SY, Wu X, Zhou MQ (2018) Effect of Electroacupuncture on Hippocampal IL-6, IL-1 $\beta$, TNF- $\alpha$ and Norepinephrine Levels in Acute Myocardial Ischemia Rats. Zhen $\mathrm{Ci}$ Yan Jiu. 43(6):365-9.

25. Yang Y, Ji L, Li G, Deng X, Cai P, Guan L (2012) Differences in thermal effects of moxibustion at Zusanli (ST 36) and Hegu (LI 4) on various facial areas in healthy people. J Traditional Chinese Med. 22(3):397-403. 


\section{ANEXO}

\section{CARTA DE CONSENTIMIENTO INFORMADO}

Lugar y fecha:

Por medio de la presente acepto participar en el protocolo de investigación titulado:

\section{Modificación de la variabilidad de la frecuencia cardíaca mediante la moxibustión en el punto de acupuntura V15}

Se me ha explicado que mi participación consistirá en: Permanecer sentado durante 5 minutos antes y 5 minutos después de moxar el punto de acupuntura $V 15$, el cual se localiza a 1.5 medidas a un lado del borde inferior de la apófisis espinosa de la quinta vértebra torácica.

Declaro que se me ha informado ampliamente sobre los posibles riesgos, inconvenientes, molestias y beneficios derivados de mi participación en el estudio, que son los siguientes.

- Enrojecimiento y inflamación de la piel después de moxar

- Sensación de sueño

- palpitaciones,

- Rara vez infección en la piel.

El investigador responsable se ha comprometido a darme información oportuna sobre cualquier procedimiento alternativo adecuado que pudiera ser ventajoso para mi tratamiento, así como a responder cualquier pregunta y aclarar cualquier duda que le plantee acerca de los procedimientos que se llevarán a cabo, los riesgos, beneficios o cualquier otro asunto relacionado con la investigación o con mi tratamiento.

Entiendo que conservo el derecho de retirarme del estudio en cualquier momento en que lo considere conveniente, sin que ello afecte la atención médica que recibo en el Instituto.

El investigador responsable me ha dado seguridades de que no se me identificará en las presentaciones o publicaciones que deriven de este estudio y de que los datos relacionados con mi privacidad serán manejados en forma 
confidencial. También se ha comprometido a proporcionarme la información actualizada que se obtenga durante el estudio, aunque esta pudiera cambiar de parecer respecto a mi permanencia en el mismo.

Nombre y firma del participante

Testigo 1

Firma:

Dom:
Testigo 2

Firma:

Dom:

Números telefónicos a los cuales puede comunicarse en caso de emergencia, dudas o preguntas relacionadas con el estudio: 
MODIFICACIÓN DE LA

VARIABILIDAD DE LA
FRECUENCIA CARDIACA MEDIANTE MOXIBUSTIÓN EN EL PUNTO DE ACUPUNTURA V15 EN SUJETOS SANOS

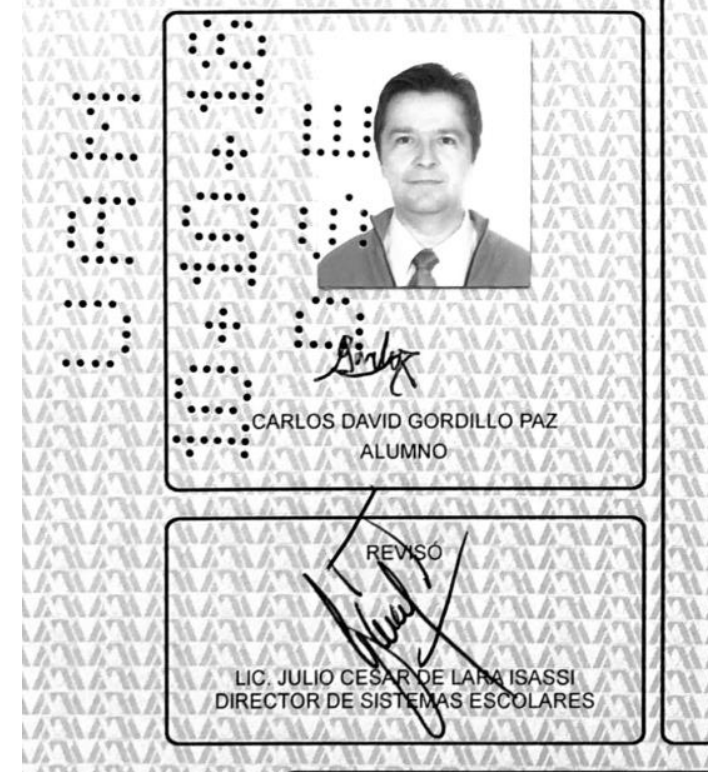

En la Ciudad de México, se presentaron a las 12:00 horas del día 8 del mes de octubre del año 2018 en la Unidad Iztapalapa de la Universidad Autónoma Metropolitana, los suscritos miembros del jurado:

DR. RUBEN ROMAN RAMOS

DR. JOSE FEDERICO RIVAS VILCHIS

DR. JOSE LUIS EDUARDO FLORES SAENZ

Bajo la presidencia del primero y con carácter de Secretario el último, se reunieron a la presentación de la Idónea Comunicación de Resultados cuya denominación aparece al margen, para la obtención del diploma de:

ESPECIALIZACION/EN ACUPUNTURA Y FITOTERAPIA

DE: CARLOS DAVID GORDILLO PAZ

y de acuerdo con el articulo 79 fracción II del Reglamento de Estudios Superiores de $\mathrm{Ia}$ Universidad Autónoma Metropolitana, los miembros del jubado resolvieron: WW

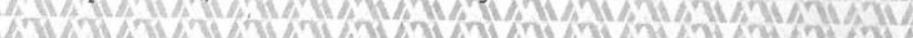

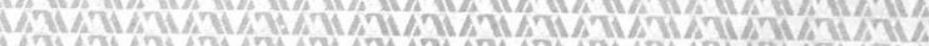

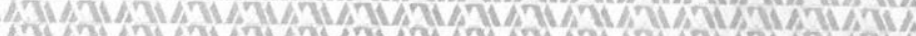

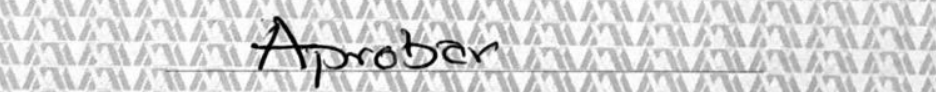

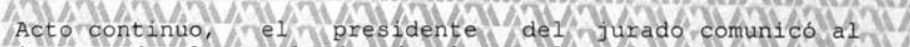
interesado el fesultado de la evaluacion y, en caso aprobatorio, le fue tomada la protesta. ming in

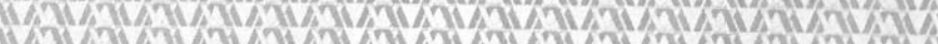

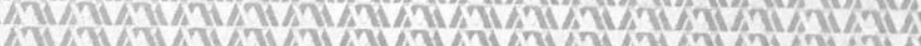
intinging ins

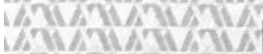

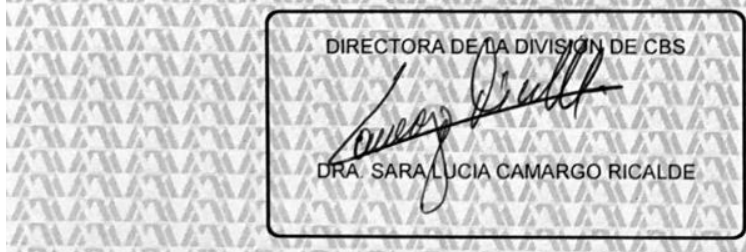
ing

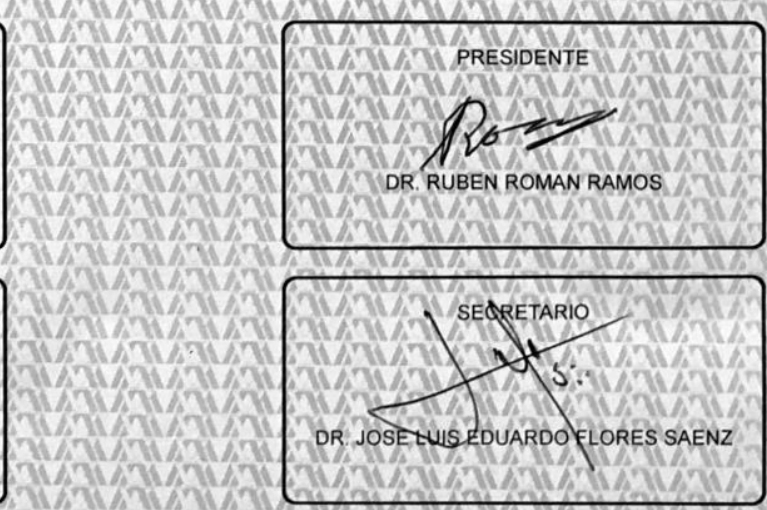

DR. JOSE FEDERICO RIVAS VILCHIS

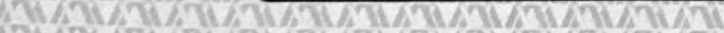

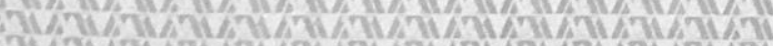

